\title{
Time Spent on Television Viewing and its Effect on Changing Values of School Going Children
}

\author{
Kalpna Arya
}

\section{INTRODUCTION}

Television is one of the source of information and among the many factors influencing values, home life television is noteworty. The popularity of television is wide spread and it has both positive as well as negative effects on children and youth depending upon the quality and contents of its programmes. Television viewing has brought about many changes in the behaviour as well as values and attitudes of the children. Sharma (1994) also studied the effect of television viewing on different values. Keeping this in mind the study was undertaken with the objective to examine the influence of television on changing values of children between 6-12 years.

\section{MATERIAL AND METHODS}

The study was conducted on children of two Govt. and two private schools in the Panchrukhi Block of Kangra district of Himachal Pradesh. One hundred fifty children (75 males and 75 females) were selected from those schools as the sample of the study. Modified Personal value questionnaire cum interview schedule (Sherry and Verma, 1994) was used in the present study to assess the effect of television on different values of children. The data were collected through personal interview method by visiting each respondent personally at home/school. The parents and teacher of children were also interviewed for collecting required information. The collected data were first tabulated on a master table from which appropriate tables were formulated depending upon the type of information required. Chi-square $\left(\chi^{2}\right)$ test was used to compute association among independent and dependent variables.

\section{RESULTS AND DISCUSSION}

Table 1 indicates the number of hours spent on television viewing by both male and female children per day. It can be seen from the table that there were only 5.33 per cent male
Table 1: Daily time spent on television watching by the respondents

\begin{tabular}{lccc}
\hline Time spent $($ hrs $)$ & $\begin{array}{c}\text { Male } \\
(n=75)\end{array}$ & $\begin{array}{c}\text { Female } \\
(n=75)\end{array}$ & $\begin{array}{c}\text { Total } \\
(N=150)\end{array}$ \\
\hline Less than 1 & $28(37.33)$ & $37(49.33)$ & $65(43.33)$ \\
$1-2$ & $32(42.67)$ & $28(37.33)$ & $60(40.0)$ \\
$2-3$ & $11(14.67)$ & $10(13.34)$ & $21(14.0)$ \\
3 and more & $4(5.33)$ & - & $4 \quad(2.67)$ \\
\hline
\end{tabular}

$\chi_{\text {Cal }}^{2}=5.560^{\mathrm{NS}}$

NS- Non- significant

Figures in parentheses indicate percentage of total sample of each aspect

respondents who watched television programmes for more than 3 hours while there were none among females. The average time spent in front of television was found to be 1 to 3 hours.

Table 2 shows the religious values of the respondents It is the family/ parents who form the habit of worship in children. No significant gender differences were found in religious values of the respondents.

Table 3 presents habits of children and their aesthetic sense i.e. appreciation of beauty etc. Most of male respondents (97.33 per cent) kept their things properly in rooms as compare to 93.33 per cent female respondents. According to the parents and teachers of the respondents, all the respondents completed their homework regularly, neatly and systematically. They also like to wear neat and clean dresses. About 52 per cent of the female respondents like to give gifts like flower pot, painting, lampshade etc. on the birthday of their friends in comparison to 64 per cent of the male respondents. Results revealed that there were difference among males and females for the same as reflected by the Chi-square value which was estimated to be significant at 5 per cent level of significance. It was also observed that there was difference among Doordarshan viewers and cable viewers regarding the aesthetic value. Females had better aesthetics values than males.

Table 4 shows that about 30 per cent of the respondents had habits of going to library. Almost 46.67 per cent of the male respondents liked to 
Table 2: Effect of television watching on religious values of the respondents

\begin{tabular}{|c|c|c|c|c|c|c|c|c|c|}
\hline \multirow{3}{*}{$\begin{array}{l}\text { Features of religious values/God/ } \\
\text { Goddess worshipped by children }\end{array}$} & \multicolumn{9}{|c|}{ Channels } \\
\hline & \multicolumn{3}{|c|}{ Doordarshan (\%) } & \multicolumn{3}{|c|}{ Cable network (\%) } & \multicolumn{3}{|c|}{$\operatorname{Total}(\%)$} \\
\hline & $\begin{array}{c}\text { Males } \\
(n=60)\end{array}$ & $\begin{array}{c}\text { Females } \\
(n=59)\end{array}$ & $\begin{array}{c}\text { Total } \\
(N=119)\end{array}$ & $\begin{array}{l}\text { Males } \\
(n=15)\end{array}$ & $\begin{array}{c}\text { Females } \\
(n=16)\end{array}$ & $\begin{array}{c}\text { Total } \\
(N=31)\end{array}$ & $\begin{array}{l}\text { Males } \\
(n=75)\end{array}$ & $\begin{array}{c}\text { Females } \\
(n=75)\end{array}$ & $\begin{array}{c}\text { Total } \\
(N=150)\end{array}$ \\
\hline \multicolumn{10}{|l|}{ A. Features of Religious Values } \\
\hline Faith in God & 98.33 & 100.00 & 99.16 & 93.33 & 87.50 & 96.77 & 97.33 & 97.33 & 97.33 \\
\hline Worship done by children & 90.67 & 100.00 & 98.32 & 86.67 & 68.75 & 77.42 & 94.67 & 93.30 & 94.00 \\
\hline Religious schedule at home & 86.67 & 84.75 & 85.71 & 80.00 & 87.50 & 83.87 & 88.00 & 85.33 & 86.67 \\
\hline Go to religious place & 100.00 & 100.00 & 100.00 & 93.33 & 75.00 & 83.87 & 98.67 & 94.67 & 96.67 \\
\hline $\begin{array}{l}\text { Like to watch religious serials } \\
\text { on television }\end{array}$ & 26.67 & 20.34 & 23.53 & 60.00 & 31.25 & 45.16 & 33.33 & 22.67 & 28.00 \\
\hline \multicolumn{10}{|c|}{ B. God/Goddess Worshipped by Children } \\
\hline Shivji & 30.00 & 30.50 & 30.25 & 80.00 & 50.00 & 64.51 & 40.00 & 34.67 & 37.33 \\
\hline Durga & 5.00 & 8.47 & 6.72 & 20.00 & 12.50 & 16.12 & 8.00 & 9.33 & 18.67 \\
\hline Krishna & 3.33 & 6.77 & 5.04 & 13.33 & 18.75 & 16.12 & 5.33 & 9.33 & 7.33 \\
\hline Ganesh & 5.00 & 1.69 & 3.36 & 13.33 & - & 6.45 & 6.67 & 1.33 & 4.00 \\
\hline Hanumaan & 5.00 & 3.38 & 4.20 & 13.33 & 12.50 & 12.90 & 6.67 & 5.33 & 6.00 \\
\hline All above & 10.00 & 16.90 & 13.44 & 28.66 & 31.25 & 29.03 & 13.33 & 20.00 & 16.67 \\
\hline Any other & 13.33 & 10.16 & 11.76 & 20.00 & 25.00 & 22.58 & 14.67 & 13.33 & 14.00 \\
\hline B) & 5 & & $\begin{array}{l}\text { A) } \chi \\
\text { B) } \chi^{2}\end{array}$ & 10 & & Cal_ & & & \\
\hline
\end{tabular}

Table 3: Effect of television watching on aesthetic values of the respondents

\begin{tabular}{|c|c|c|c|c|c|c|c|c|c|}
\hline \multirow{3}{*}{$\begin{array}{l}\text { Features of aesthetic values/ } \\
\text { Free time activities }\end{array}$} & \multicolumn{9}{|c|}{ Channels } \\
\hline & \multicolumn{3}{|c|}{ Doordarshan (\%) } & \multicolumn{3}{|c|}{ Cable network (\%) } & \multicolumn{3}{|c|}{ Total (\%) } \\
\hline & $\begin{array}{l}\text { Males } \\
(n=60)\end{array}$ & $\begin{array}{c}\text { Females } \\
(n=59)\end{array}$ & $\begin{array}{c}\text { Total } \\
(N=119)\end{array}$ & $\begin{array}{l}\text { Males } \\
(n=15)\end{array}$ & $\begin{array}{c}\text { Females } \\
(n=16)\end{array}$ & $\begin{array}{c}\text { Total } \\
(N=31)\end{array}$ & $\begin{array}{l}\text { Males } \\
(n=75)\end{array}$ & $\begin{array}{c}\text { Females } \\
(n=75)\end{array}$ & $\begin{array}{c}\text { Total } \\
(N=150)\end{array}$ \\
\hline \multicolumn{10}{|l|}{ A. Features of Aesthetic Values } \\
\hline $\begin{array}{l}\text { Keep books copies clothes } \\
\text { andshoes properly }\end{array}$ & 98.33 & 98.30 & 98.31 & 93.33 & 75.00 & 83.87 & 97.33 & 93.33 & 95.33 \\
\hline $\begin{array}{l}\text { Complete home work regularly } \\
\text { neatly and systematically }\end{array}$ & 100.00 & 100.00 & 100.00 & 100.00 & 100.00 & 100.00 & 100.00 & 100.00 & 100.00 \\
\hline $\begin{array}{l}\text { Like to wear neat and } \\
\text { clean dresses }\end{array}$ & 100.00 & 100.00 & 100.00 & 100.00 & 100.00 & 100.00 & 100.00 & 100.00 & 100.00 \\
\hline Gift to friends & 48.33 & 67.79 & 57.98 & 66.67 & 50.00 & 58.06 & 52.00 & 64.00 & 58.00 \\
\hline $\begin{array}{l}\text { God is present in all } \\
\text { beautiful things }\end{array}$ & 91.67 & 98.30 & 94.95 & 60.00 & 68.75 & 64.51 & 85.33 & 92.00 & 88.67 \\
\hline \multicolumn{10}{|l|}{ B. Free Time Activities } \\
\hline Drawing/painting & 20.00 & 28.33 & 20.16 & 33.33 & 37.50 & 35.48 & 22.67 & 24.00 & 28.33 \\
\hline Listening music & 8.33 & 3.38 & 5.88 & 40.00 & 18.75 & 29.03 & 14.67 & 6.67 & 10.67 \\
\hline Dancing & 6.67 & 3.38 & 5.04 & 20.00 & 6.25 & 12.90 & 9.33 & 4.00 & 6.67 \\
\hline Going outside & 28.33 & 16.94 & 22.68 & 53.33 & 56.25 & 54.83 & 33.33 & 25.33 & 29.33 \\
\hline Gardening & 6.67 & 1.69 & 4.20 & - & - & 16.12 & 15.33 & 1.33 & 3.33 \\
\hline Watching television & 20.00 & 3.38 & 11.76 & 93.33 & 6.25 & 48.38 & 34.67 & 4.00 & 19.33 \\
\hline Above all activities & 1.67 & - & 0.84 & - & - & 3.22 & 1.33 & - & 10.67 \\
\hline Other activities & 5.00 & 3.38 & 4.20 & 13.33 & 18.75 & 16.12 & 6.67 & 6.67 & 6.67 \\
\hline $\begin{array}{l}\text { NS }=\text { Non- significant } \\
* \text { Significant at } 5 \% \text { level of sig }\end{array}$ & \multicolumn{3}{|c|}{$\begin{array}{l}\text { A) } \chi_{\text {Cal }}^{2}=1.634^{\mathrm{NS}} \\
\text { B) } \chi_{\text {Cal }}^{2}=6.299^{\mathrm{NS}}\end{array}$} & \multicolumn{3}{|c|}{$\begin{array}{l}\text { A) } \chi^{2}=1.879^{\mathrm{NS}} \\
\text { B) } \chi_{\mathrm{Cal}}^{2}=10.567^{\mathrm{NS}}\end{array}$} & \multicolumn{3}{|c|}{$\begin{array}{l}\text { A) } \chi_{\mathrm{Cal}}^{2}=1.000^{\mathrm{NS}} \\
\text { B) } \chi_{\mathrm{Cal}}^{2}=15.167^{*}\end{array}$} \\
\hline
\end{tabular}

read extra books in comparison to almost 29.33 per cent of females. About 73.33 per cent of the females and 62.67 per cent of males felt that educated families were good families. Only 33.33 per cent of the male respondents liked to miss school for the sake of television/cinema. Whereas females did not miss school. About 91 per cent of the respondents had interest in learning new things. Chi-square value was estimated to be highly significant at 1 per cent level of significance and 5 Per cent level of significance. Table also shows the qualities of a good teacher according to the respondents. Results also revealed that knowledge value of children increases due to television and males had better knowledge value than females, whether they 
Table 4 Effect of television watching on knowledge values of the respondents

\begin{tabular}{|c|c|c|c|c|c|c|c|c|c|}
\hline \multirow{3}{*}{$\begin{array}{l}\text { Features of knowledge values/ } \\
\text { Qualities of good teacher }\end{array}$} & \multicolumn{9}{|c|}{ Channels } \\
\hline & \multicolumn{3}{|c|}{ Doordarshan (\%) } & \multicolumn{3}{|c|}{ Cable network (\%) } & \multicolumn{3}{|c|}{ Total (\%) } \\
\hline & $\begin{array}{l}\text { Males } \\
(n=60)\end{array}$ & $\begin{array}{c}\text { Females } \\
(n=59)\end{array}$ & $\begin{array}{c}\text { Total } \\
(N=119)\end{array}$ & $\begin{array}{l}\text { Males } \\
(n=15)\end{array}$ & $\begin{array}{l}\text { Females } \\
(n=16)\end{array}$ & $\begin{array}{c}\text { Total } \\
(N=31)\end{array}$ & $\begin{array}{l}\text { Males } \\
(n=75)\end{array}$ & $\begin{array}{c}\text { Females } \\
(n=75)\end{array}$ & $\begin{array}{c}\text { Total } \\
(N=150)\end{array}$ \\
\hline \multicolumn{10}{|l|}{ A. Features of Knowledge Values } \\
\hline Visit to library & 30.00 & 39.33 & 27.73 & 33.33 & 43.75 & 38.71 & 30.67 & 29.33 & 30.00 \\
\hline Read extra & 40.00 & 35.59 & 37.82 & 40.00 & 37.50 & 38.71 & 46.67 & 29.33 & 38.00 \\
\hline Educated family is good family & 75.00 & 71.19 & 73.11 & 53.33 & 43.75 & 48.39 & 73.33 & 62.67 & 68.00 \\
\hline $\begin{array}{l}\text { Miss the school for television/ } \\
\text { cinema }\end{array}$ & 20.00 & - & 10.08 & 86.67 & - & 41.94 & 33.33 & - & 16.67 \\
\hline Interest in learning new things & 96.67 & 98.30 & 97.48 & 80.00 & 56.25 & 67.74 & 93.33 & 89.33 & 91.33 \\
\hline Belief in hard work & 100.00 & 100.00 & 100.00 & 100.00 & 100.00 & 100.00 & 100.00 & 100.00 & 100.00 \\
\hline \multicolumn{10}{|l|}{ B. Qualities of Good Teacher } \\
\hline Soft spoken & 30.00 & 35.59 & 32.77 & 33.33 & 25.00 & 29.03 & 30.67 & 33.33 & 32.00 \\
\hline Having more knowledge & 3.33 & 5.08 & 4.20 & 13.33 & 12.50 & 12.90 & 5.33 & 6.67 & 16.00 \\
\hline Intelligent & 20.00 & 8.47 & 14.29 & 40.00 & 18.75 & 29.03 & 24.00 & 10.67 & 17.33 \\
\hline Tell about new things & 3.33 & 1.69 & 2.52 & 6.67 & - & 3.23 & 4.00 & 1.33 & 2.67 \\
\hline Above all qualities & 21.67 & 16.95 & 19.33 & 33.33 & 12.50 & 22.58 & 24.00 & 16.00 & 20.00 \\
\hline Other qualities & 18.33 & 18.64 & 18.49 & 46.67 & 25.00 & 35.48 & 24.00 & 20.00 & 22.00 \\
\hline $\begin{array}{l}\text { NS= Non- significant } \\
* * \text { Significant at } 1 \% \text { level of signi } \\
* \text { Significant at } 5 \% \text { level of signifi }\end{array}$ & & \multicolumn{2}{|c|}{$\begin{array}{l}\text { A) } \chi_{{ }_{\mathrm{Cal}}^{2}}^{2}=11.442^{*} \\
\text { B) } \chi_{\mathrm{Cal}}=3.603^{\mathrm{NS}}\end{array}$} & \multicolumn{3}{|c|}{$\begin{array}{l}\text { A) } \chi_{\mathrm{Cal}}^{2}=12.197^{*} \\
\text { B) } \chi_{\mathrm{Cal}}^{2}=1.362^{\mathrm{NS}}\end{array}$} & \multicolumn{3}{|c|}{$\begin{array}{l}\text { A) } \chi_{{ }^{C a l}}^{2}=24.061^{*} * \\
\text { B) } \chi_{\mathrm{Cal}}^{2}=4.417^{\mathrm{NS}}\end{array}$} \\
\hline
\end{tabular}

were cable network viewers or Doordarshan viewers.

In general, sex differences were not very sharp regarding, democratic, social economic hedonistic and health values of children. There were not much differences among males and females regarding religious values but females were slightly more religious than males. Females had better aesthetic values while males had better knowledge values than females.

KEYWORDS Democratic. Social. Economic. Health. Sex Differences. Television

ABSTRACT The present study was undertaken to examine the effect of television on different selected values of children in the age group of 6-12 years. This study was conducted in the Panchrukhi Block of Kangra District of Himachal Pradesh. A total of hundred fifty children (75 each from Govt. and Private schools) of both sexes were selected randomly for this study. The data were collected by personal contact on different television viewing habits of children by using a questionnaire cum interview schedule. The effect of television on different selected values of children was assessed by using Chi-square statistic analysis. Results showed that sex differences were not apparent regarding democratic, Social, economic, hedonistic and health values of children and sex differences were observed among religious, aesthetic and knowledge values.

\section{REFERENCES}

Anonymous: The effects of television on the pro-social behaviour of young children. Young Children, 32: 26 (1977).

Brock, Antony: How television affects children.Patriot Weekly Magzine, 8(353): 1-2 (1971).

Gaber, Peter: Developing the child and youth care profession through affective use of media. Child Youth Services, 13(1): 199-213 (1990).

Gold Smith, Elaine:Difference in reciprocal peer social relations among children who view low, moderate and high amounts of television. Journal of Home Economics, 16(4): 207-214 (1987).

Kaur, Jasraj: Impact of viewing T.V. on the social interaction of rural illiterate neo-literate adults. Journal of Psycholingua, 31(2): 115-120 (2001).

Ramarethinam and Preethi, R.: Social and cultural impact of satellite television. Kisan World, 55-56 (2001).

Selvon: Television Programmes and Changing Values of Different Age Groups. M.Sc. thesis submitted to Nagpur University, Nagpur (1982).

Sharma, A.: Television Programmes and Changing Values of Different Age Group. M.Sc. thesis, Nagpur University, Nagpur ( 1994-95).

Singh, S. and Arora, L: The effect of television viewing upon youth of Doon valley. Indian Journal of Psychological Review, 38(9-10): 21-26 (1992).

Thakur, Yama and C.P.Khokhar: Mass media and children. Journal of Psycholingua, 31(3 ): 135-38 (2001).

Author's Address: Kalpna Arya, Assistant Extension Specialist (H.Sc.) KVK, Mandi 175001, Himachal Pradesh, India

Address for Correspondence: Dr. Kalpna Arya, Assistant Extension Specialist (H.Sc.), Prem Niwas, Kansa Chowk P.O. Dhaban Teh Sadar, District Mandi 175 027, Himachal Pradesh, India 